SHORT REPORT

\title{
Interferon beta in multiple sclerosis: experience in a British specialist multiple sclerosis centre
}

\author{
B D Dubois, E Keenan, B E Porter, R Kapoor, P Rudge, A J Thompson, D H Miller, \\ G Giovannoni
}

J Neurol Neurosurg Psychiatry 2003;74:946-949

Background: The efficacy of interferon beta (IFN beta) is well established in relapsing-remitting multiple sclerosis (MS). However, the use of this drug in clinical practice is complex, especially because it is only partially effective, its long term efficacy and side effects are unknown, its efficacy may be abrogated by the development of neutralising antibodies, compliance is variable, and its cost effectiveness is controversial.

Objectives and Methods: Analysis of a prospectively followed up series of 101 MS patients treated with IFN beta was undertaken to: (1) monitor the outcome of IFN beta treatment in clinical practice; (2) compare the immunogenicity of the three commercial IFN beta preparations available; (3) assess the proportion of patients fulfilling the current guidelines of the Association of British Neurologists for stopping IFN beta therapy.

Results: During a median treatment period of 26 months (range 2-85), the relapse rate decreased by $41 \%$. Although the reduction in the relapse rate was similar for all three commercial products, none of the Avonex treated patients were relapse free, compared with 19\% of the Betaferon treated and $27 \%$ of the Rebif treated patients ( $p=0.02)$. Neutralising antibodies were not detected in Avonex treated patients (0 of 18), compared with 12 of $32(38 \%)$ Betaferon treated and 10 of $23(44 \%)$ Rebif treated patients $(p=0.02)$. Forty of 101 (40\%) patients satisfied the current (2001) Association of British Neurologists criteria for stopping IFN beta treatment at some stage during their treatment.

Conclusion: IFN beta is effective in reducing the relapse rate in patients with relapsing-remitting MS in routine clinical practice. However, after a median treatment duration of 26 months, $40 \%$ of initially relapsing-remitting MS patients seem to have ongoing disease activity, presenting as disabling relapses or insidious progression.

$\mathrm{T}$ hree large, multicentre, randomised, double blind, placebo controlled trials have demonstrated that interferon beta (IFN beta) reduces the relapse rate in patients with relapsing-remitting multipe sclerosis $(\mathrm{MS})^{1-3}$ and in two of these studies IFN beta slowed the rate of accumulation of disability. ${ }^{2}$ One of the questions that has not been answered by these trials, is for how long IFN beta treatment should continue. This issue is important, not only to prevent unnecessary treatment, but to allow patients access to other potentially efficacious therapies and to enable health authorities to plan drug provision for MS.

This prospective audit describes the implementation of IFN beta therapy at a tertiary referral centre, the National Hospital for Neurology and Neurosurgery (NHNN) in London. The unblinded, observational concept carries a number of inherent limitations, such as drug selection criteria, potential unblinding effects, and patient selection criteria.
The aims of the audit were therefore (1) to evaluate the efficacy of IFN beta treatment in clinical practice; (2) to establish the immunogenicity of the different products and correlate the occurrence of neutralising antibodies (NABs) with clinical parameters; (3) to assess "failure of treatment" as defined by the recently published guidelines from the Association of British Neurologists (ABN).

\section{METHODS \\ Patients}

An analysis was performed on all patients with relapsingremitting MS who started IFN beta therapy before I January 2000 at the NHNN in London. Only patients who experienced at least two clinically significant relapses during the previous two years and had an Expanded Disability Status Scale (EDSS) score of less than 5.5 were given IFN beta. A minimum follow up period of at least 12 months at the NHNN was required to be included in the audit.

\section{Demographic and efficacy parameters}

The following parameters were assessed during the analysis: age at onset of disease, duration of disease, duration of treatment, and the IFN beta product. Disease course, relapse rate, time of relapse, corticosteroid use, and mobility were evaluated before treatment and at the end of the assessment period. Patients were classified as having secondary progressive disease only if there was a clear progression in at least the preceding six months without the interference of episodes. The annualised relapse rate and use of corticosteroids in the two years before treatment were compared with the respective rates on treatment. Relapses were defined as disabling if they required corticosteroid administration. Disability was not formally assessed with the EDSS but was prospectively scored using the following simple mobility scale: $0=$ asymptomatic; $\mathrm{l}=$ able to walk unaided for more than $500 \mathrm{~m} ; 2=$ able to walk unaided for less than $500 \mathrm{~m} ; 3$ =walking with unilateral support; $4=$ walking with bilateral support; $5=$ needs wheelchair outdoors; $6=$ wheelchair bound

The proportion of patients fulfilling the current guidelines of the ABN for stopping IFN beta therapy was also assessed. The ABN considers the following features as likely to indicate a lack of treatment efficacy:

(1) two disabling relapses, as defined by the examining neurologist, within a 12 months period

(2) development of secondary progressive MS

(3) loss of ability to walk, with or without assistance, persistent for at least six months

Abbreviations: IFN beta, interferon beta; MS, multiple sclerosis; NAB, neutralising antibody; EDSS, Expanded Disability Status Scale; $A B N$, Association of British Neurologists 
Table 1 Demographic and efficacy data of relapsing-remitting MS patients, treated with interferon beta for at least 12 months

\begin{tabular}{|c|c|c|c|c|}
\hline Parameters & All products & Avonex & Betaferon & Rebif \\
\hline Number of patients & $n=101$ & $n=24$ & $n=37$ & $n=37$ \\
\hline \multicolumn{5}{|l|}{ Demographics } \\
\hline age at onset of disease (y) & $27.6(7.0)$ & $27.4(6.9)^{*}$ & $26.6(6.3)^{*}$ & $29.2(8.0)^{*}$ \\
\hline duration of disease $(y)$ & $9.5(6.3)$ & $9.0(4.8)^{*}$ & $9.5(5.7)^{*}$ & $10.0(7.8)^{*}$ \\
\hline duration of treatment $(\mathrm{m})$ & $29.4(13.3)$ & $29.8(8.2) \dagger$ & $38.2(12.6) \dagger$ & $19.2(3.6) \dagger$ \\
\hline \multicolumn{5}{|l|}{ Efficacy } \\
\hline relapse rate at baseline & $1.9(0.9) \dagger$ & $2.2(1.3)^{*}$ & $1.9(0.7)^{*}$ & $1.9(0.7)^{*}$ \\
\hline relapse rate on treatment & $1.2(1.2) \dagger$ & $1.3(1.1)^{*}$ & $1.2(1.4)^{*}$ & $1.1(0.9) *$ \\
\hline relapse free & $16 / 96(16.7 \%)$ & $0 / 22 \ddagger$ & $7 / 37(19 \%) \ddagger$ & $10 / 37(27 \%) \ddagger$ \\
\hline corticosteroid use at baseline & $0.9(0.7) \dagger$ & $1.1(0.8)^{*}$ & $0.9(0.6)^{*}$ & $0.8(0.7)^{*}$ \\
\hline corticosteroid use on treatment & $0.6(1.0) \dagger$ & $0.7(1.2)^{*}$ & $0.6(1.2)^{*}$ & $0.6(0.8)^{*}$ \\
\hline mobility at baseline & $1.4(1) \dagger$ & $1.4(0.9)^{*}$ & $1.4(0.9)^{*}$ & $1.4(1.0) *$ \\
\hline mobility on treatment & $2.1(1.6) \dagger$ & $2.2(1.7)^{*}$ & $2.1(1.7)^{*}$ & $2.0(1.6)^{*}$ \\
\hline SP on treatment & $23 / 101(22.8 \%)$ & $7 / 24(29.2 \%)$ & $9 / 37(24.3 \%)$ & $6 / 37(16.2 \%)$ \\
\hline early discontinuation & 5 & 2 & 3 & 0 \\
\hline \multicolumn{5}{|l|}{ Neutralising antibodies } \\
\hline Positive & $22 / 73(30.1 \%)$ & $0 / 18$ & $12 / 32(38 \%)$ & $10 / 23(44 \%)$ \\
\hline \multicolumn{5}{|l|}{ ABN stopping criteria } \\
\hline relapses or secondary progression & $40 / 101(39.6 \%)$ & $10 / 24(41.7 \%)$ & $16 / 37(43.2 \%)$ & $13 / 37(35.1 \%)$ \\
\hline relapses & $17 / 101(16.8 \%)$ & $3 / 24(12.5 \%)$ & $7 / 37(18.9 \%)$ & $7 / 37(18.9 \%)$ \\
\hline secondary progression & $15 / 101(14.9 \%)$ & $4 / 24(16.7 \%)$ & $6 / 37(16.2 \%)$ & $4 / 37(10.8 \%)$ \\
\hline relapses and secondary progression & $8 / 101(7.9 \%)$ & $3 / 24(12.5 \%)$ & $3 / 37(8.1 \%)$ & $2 / 37(5.4 \%)$ \\
\hline inability to walk & $2 / 101$ (2\%) & $1 / 24(4.2 \%)$ & $1 / 37(2.7 \%)$ & $0 / 24(0 \%)$ \\
\hline
\end{tabular}

\section{Neutralising anti-IFN beta antibodies}

Neutralising anti-IFN beta-la and beta-lb antibodies were tested for in serum of patients in whom blood samples were taken at baseline and one year after treatment onset using a standard cytopathic effect assay in an independent laboratory. ${ }^{4}$ Samples were coded and assayed blind.

\section{Statistical analysis}

Means and standard deviations of the mean were calculated for relapse rate, corticosteroid use, and mobility before and on treatment as well as on the absolute and relative differences of these parameters before treatment and at the end of the assessment period. The means of the duration of disease, the duration of treatment, and the age at onset of disease were also analysed. Statistical analysis on these mean values was performed using a paired sample or an independent samples $t$ test. Fisher's exact test was used to compare numbers of relapse free patients in various subgroups. Differences between products were assessed by one way analysis of variance. Influence of NABs on number of relapse free patients or patients becoming secondary progressive was assessed using the $\chi^{2}$ statistical method. Results were considered statistically significant if $\mathrm{p}<0.05$.

\section{RESULTS}

The data concerning demographics, treatment efficacy, NABs, and stopping criteria are shown in table 1.

\section{Patients}

One hundred and one patients (67 women, 34 men) were started on IFN beta before 1 January 2000 and were followed up at the NHNN for at least 12 months. The median duration of treatment was 26 months (range 12-85). Five patients stopped treatment: two because of pregnancy and three (one taking Avonex, two taking Betaferon) because of lack of efficacy and persistent side effects. Five patients changed product during the assessment period. They were included in the general analysis, but not in the analyses that involved product comparisons.

\section{Treatment efficacy}

Relapse rate and corticosteroid use were significantly reduced after treatment with IFN beta. However, mobility worsened on the mobility scale $(\mathrm{p}<0.001)$.
Twenty three patients $(22.8 \%$, six taking Avonex, eight taking Betaferon, six taking Rebif, and three who changed products) developed secondary progressive disease by the end of the assessment period. No significant differences were found between the products in regard to their effect on relapse rate, corticosteroid use, and mobility. However, the number of relapse free patients differed significantly between the various products. None of the Avonex treated patients (0 of 22) was relapse free whereas seven of 37 (19\%) Betaferon and 10 of 37 $(27 \%)$ Rebif treated patients did not experience relapses during the treatment period $(\mathrm{p}=0.02)$ (fig $\mathrm{l}$ ).

\section{Neutralising anti-IFN beta antibodies \\ General analysis}

NAB test results after one year of IFN beta therapy were available in 73 (48 female, 25 male) of the 101 relapsing-remitting patients. Anti-IFN beta antibodies were found in $22(30 \%)$ of these 73 patients. In three $(4 \%$ ) patients (one taking Betaferon, two taking Rebif) the antibodies were not cross reactive between the IFN beta-lb and beta-la preparations. The means of the various demographic and clinical data were compared in antibody negative versus antibody positive patients. No significant differences were found for relapse rate, corticosteroid use, and mobility at baseline and at the end of

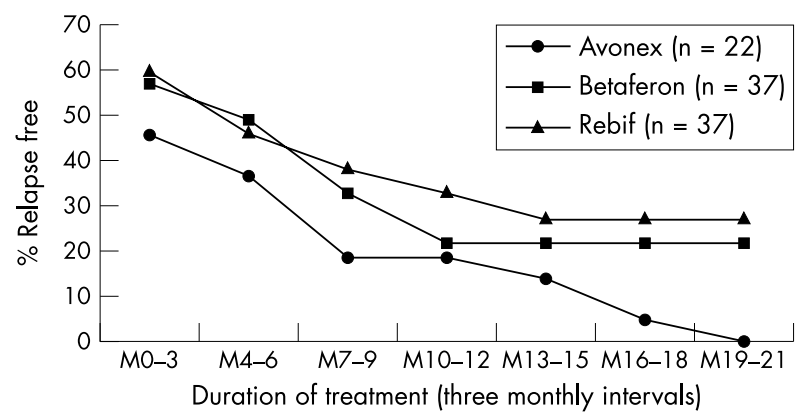

Figure 1 This figure shows the proportion of relapse free patients according to the type of IFN beta treatment in three monthly intervals. Significantly fewer Avonex treated patients were relapse free at months 16 to 18 and months 19 to 21 than Betaferon treated and Rebif treated patients. 
the assessment period, relative and absolute changes in relapse rate, corticosteroid use, and mobility. The presence of antibodies did not change the number of relapse free patients or the number of patients becoming secondary progressive.

\section{Comparison of agents}

None of the Avonex treated patients developed antibodies $(0$ of 18), whereas $12(38 \%)$ of the Betaferon treated and 10 $(44 \%)$ of the Rebif treated patients did. When antibody positive $(n=12)$ and antibody negative $(n=20)$ patients taking Betaferon were compared, a trend $(p=0.08)$ towards a higher reduction in relative relapse rate was seen in antibody negative patients (antibody negative 56\% v antibody positive 20\%). Antibody negative patients were not more often relapse free. Similar analyses were performed in antibody positive $(n=10)$ and antibody negative $(n=13)$ patients who were treated with Rebif and no significant differences were found regarding the efficacy parameters.

Treatment failure according to the $\mathrm{ABN}$ stopping criteria Of the 101 relapsing-remitting MS patients, 40 (40\%) fulfilled the current ABN criteria for stopping therapy during the audit. Eight (8\%) patients met both the first two stopping criteria (two disabling relapses within a 12 month period and the subsequent development of secondary progression), 15 (15\%) became secondary progressive (two of these patients also fulfilled the third stopping criterion), and 17 (17\%) had at least two disabling (for example, requiring corticosteroids) relapses within a 12 month period. In 24 of 25 of these patients with two disabling relapses within a 12 month period, these occurred within 18 months of starting treatment. The change in relapse frequencies during these first 18 months of the treatment was a relatively good predictor for the subsequent follow up period. However, despite fulfilling the ABN stopping criteria, six (24\%) of the 25 patients with at least two disabling relapses had a reduced relapse rate on treatment compared with the two years before starting treatment. On the other hand, five of 101 patients who did not fulfill the ABN stopping criteria had an increased relapse frequency compared with the two years before starting treatment, although these relapses were non-disabling.

\section{DISCUSSION}

Although the indication for using IFN beta in relapsingremitting MS is based on large placebo controlled clinical trials, ${ }^{1-3}$ treatment of patients with this disease modifying agent should be reviewed in clinical practice. Reasons for this include the partial effect of the drug, the difficulty in distinguishing "responders" from "non-responders", the unknown long term efficacy and side effects, and the high cost of the therapy. This audit reports the NHNN's experience from the first four years of IFN beta therapy in MS. It must be emphasised that this was an unblinded observational audit with a comparatively small number of patients, particularly for the subgroup analyses.

The results on efficacy of IFN beta treatment in our patients are largely consistent with the published trial data.

Within the limitations that the number of patients was small and treatment not being randomly allocated, all three agents seemed to reduce relapse frequency to a similar extent. A proportion of patients taking the more frequent doses of the subcutaneous preparations became relapse free (fig l). This is in accordance with recent comparative studies ${ }^{55}$ The preparations differed in their immunogenic profile. NABs were found in none of the Avonex treated patients, whereas 38\% of the Betaferon treated, and $44 \%$ of Rebif treated patients were positive. This difference in the immunogenicity is in keeping with previously reported data. ${ }^{6-10}$ It has recently been demonstrated that NABs reduce IFN beta's therapeutic effect but this only became apparent after four years of observation. ${ }^{11}$ This may explain why no clinical effects of NABs were noted in our audit. Interestingly, a trend was seen in patients on Betaferon who had received IFN beta the longest.
Finally, although IFN beta therapy was only stopped because of apparent lack of efficacy in 3\% of patients, $40 \%$ of our patients would have fulfilled the current ABN criteria for stopping therapy. More than half of those $(62.5 \%)$ had at least two disabling relapses in 12 months and $57.5 \%$ developed secondary progressive disease, with or without superimposed relapses. The time point at which the disabling relapse criterion was fulfilled demonstrates that the treatment response with regard to disabling relapses might be evaluated after as little as 18 months of IFN beta therapy. Analysis of the disease course of the 25 patients who fulfill the ABN criteria on relapses for stopping therapy highlight important features that should be taken into account. Of these 25 patients, 10 $(40 \%)$ experienced a lower or equal number of relapses compared with baseline. On the other hand, five of 101 relapsingremitting MS patients who had a higher relapse rate on treatment than at baseline, did not fulfill the ABN stopping criteria. These data question the current wisdom of defining a stopping criterion based solely on the number of disabling relapses. This will be difficult to implement on an individual patient basis particularly as IFN beta therapy is widely acknowledged to being only a partially effective therapy. It would be more appropriate to include both the baseline relapse rate and the severity of relapses in formulating a relapse based stopping criterion. In contrast, conversion to secondary progressive MS, which is more difficult to evaluate than relapses, may be a more practical stopping criterion to implement, particularly as IFN beta treatment does not show a robust effect in patients with secondary progressive MS. ${ }^{12-15}$ The future evaluation of the appropriateness of stopping criteria may be assisted by using objective predefined markers of disease activity or progression, or both. Examples of these could include magnetic resonance imaging parameters or NABs.

\section{ACKNOWLEDGEMENTS}

The authors thank $\mathrm{H}$ Watt for statistical advice. B Dubois was a research assistant of the Fund for Scientific Research (FWOVlaanderen) and was supported by the Scientific Research for Multiple Sclerosis (Wetenschappelijk Onderzoek Multiple Sclerose, WOMS, Belgium) and by a Glaxo-Smith-Kline Grant in Neurology.

\section{Authors' affiliations \\ B D Dubois, E Keenan, B E Porter, R Kapoor, P Rudge,}

A J Thompson, D H Miller, G Giovannoni, Institute of Neurology and National Hospital for Neurology and Neurosurgery, London, UK

Competing interests: DB, EK, BP, PR, RK, AJT, DHM, and GG have participated in meetings sponsored by, and received honorariums from, pharmaceutical companies marketing treatments for multiple sclerosis; our departments have received financial support for participation in randomised controlled trials of IFNbeta-1 b, IFNbeta-l a and copaxone in multiple sclerosis (Schering, Biogen, and Teva); PR, DHM, and GG have received honorariums for acting in an ad hoc capacity as advisors to various pharmaceutical companies who have drug development programmes for multiple sclerosis.

Correspondence to: Dr G Giovannoni, Department of

Neuroinflammation, Institute of Neurology, Queen Square, London WC1N 3BG, UK; G.Giovannoni@ion.ucl.ac.uk

Received 6 February 2002

Accepted in final revised form 28 January 2003

\section{REFERENCES}

1 The IFNB Multiple Sclerosis Study Group. Interferon beta- $1 \mathrm{~b}$ is effective in relapsing-remitting multiple sclerosis: I. Clinical results of a multicenter, randomized, double-blind, placebo-controlled trial. Neurology 1993:43:655-61.

2 Jacobs LD, Cookfair DL, Rudick RA, et al. Intramuscular interferon beta-l a for disease progression in relapsing multiple sclerosis. Ann Neurol 1996:39:285-94.

3 PRISMS Study Group. Randomised double-blind placebo-controlled study of interferon $\beta$-la in relapsing/remitting multiple sclerosis. Lancet 1998:352:1498-504 
4 Antonelli G, Bagnato F, Pozzilli C, et al. Development of neutralizing antibodies in patients with relapsing-remitting multiple sclerosis treated with IFN-betala. J Interferon Cytokine Res 1998;18:345-50.

5 Durelli L, Verdun E, Barbero P, et al. Every-other-day interferon beta- $1 \mathrm{~b}$ versus once-weekly interferon beta-la for multiple sclerosis: results of a 2 -year prospective randomised multicentre study (INCOMIN). Lancet 2-year prospective rand

2002;359: 1453-60.
5a Panitch H, Goodin DS, Francis G, et al. Randomised, comparative study of interferon beta- la treatment regimens in MS: The EVIDENCE Trial. Neurology 2002;59:1496-506.

6 Perini $\mathbf{P}$, Facchinetti A, Bulian $P$, et al. Interferon-beta (INF-beta) antibodies in interferon-betala and interferon beta- 1 b-treated multiple sclerosis patients. Prevalence, kinetics, cross-reactivity, and factors enhancing interferon-beta immunogenicity in vivo. Eur Cytokine Netw $2001 ; 12: 56-61$.

7 Ross C, Clemmesen KM, Svenson M, et al. Immunogenicity of interferon-beta in multiple sclerosis patients: influence of preparation, dosage, dose frequency and route of administration. Ann Neurol 2000;48:706-12.

8 Khan OA, Dhib-Jalbut SS. Neutralizing antibodies to interferon beta-1a and interferon beta-1b are cross-reactive. Neurology 1998;51:1698-702.
9 Rudick RA, Simonian NA, Alam JA, et al. Incidence and significance of neutralizing antibodies to interferon beta-la in multiple sclerosis. neutralizing antibodies to interfer
Neurology 1998:50:1266-72.

10 Fernandez O, Mayorga C, Luque G, et al. Study of binding and neutralising antibodies to interferon-beta in two groups of relapsing-remitting multiple sclerosis patients. J Neurol 2001;248:383-8.

11 The PRISMS Study Group and the University of British Columbia MS/MRI Analysis Group. PRISMS-4: Long-term efficacy of interferon- $\beta$-1 a in relapsing MS. Neurology 2001;56:1628-36.

12 European Study Group on interferon $\beta-1 \mathrm{~b}$ in Secondary Progressive MS. Placebo-controlled multicentre randomised trial of Progressive MS. Placebo-controlled multicentre randomised trial of
interferon $\beta-1 \mathrm{~b}$ in treatment of secondary progressive multiple sclerosis. interferon $\beta-1 b$ in treatment
Lancet 1998:352:1491-7.

13 SPECTRIMS Study Group. Randomized controlled trial of interferon-beta-la in secondary progressive MS: clinical results. Neurology 2001;56:1496-504.

14 Goodkin DE, and the North American Study Group on Interferon beta- $1 \mathrm{~b}$ in secondary progressive MS. Interferon beta- $1 \mathrm{~b}$ in secondary progressive MS: clinical and MRI results of a 3-year randomized controlled trial. Neurology 2000;54:2352

15 Cohen JA, Goodman AD, Heidenreich FR, et al. Results of IMPACT, a phase 3 trial of interferon beta-la in secondary progressive multiple sclerosis. Neurology 2001;56:S20.003

\section{HISTORICAL NOTE}

\section{Sir James Crichton-Browne (1840-1938)}

D mes Crichton-Browne ${ }^{1}$ was born in Edinburgh, the son of Dr WAF Browne, first superintendent of Crichton Royal, Dumfries. James was educated in Dumfries and began his medical studies at Edinburgh University in 1857, a pupil of Joseph Lister (1827-1912) and James Syme (1799-1879).

His interests in psychiatry were soon evident. As a medical student he read a paper to the Royal Medical Society, The psychical diseases of early life. Crichton-Browne graduated in 1861, and obtained the MD in 1862. He worked in asylums in Derby, Devon, and Newcastle. In 1866, at the early age of 26, he was appointed as Superintendent Medical Director of the West Riding Lunatic Asylum at Wakefield, and in nine years established the hospital as a leading centre of research and treatment. Though lacking Ferrier's scientific ability, he was a skilled administrator and a flamboyant highly persuasive speaker. He inaugurated the Annual medical reports of the West Riding Asylum in 1871, which were published annually for six years, and 62 of these 79 articles came from Wakefield Asylum. And he appointed a pathologist, the first to occupy a research laboratory within an asylum. He attracted many talented young men to cooperate in his research. They included David Ferrier and Hughlings Jackson, who worked on cerebral localisation, and on epilepsy. ${ }^{2}$ They painstakingly studied clinical phenomena and the neuropathology of their patients. For instance, in general paralysis of the insane, over 1500 autopsies were performed. In search of therapies, CrichtonBrowne also experimented with the effects of electrical stimulation of the cranium (analogous to ECT) and investigated the effects of ergot, nitrous oxide, and opium. One can understand the high reputation of their medical reports of the West Riding Asylum. ${ }^{3}$ In 1878, Crichton-Browne with Ferrier, Jackson, and Sir John Bucknill founded the celebrated neurological journal Brain. By now the doyen of mental health, Crichton-Browne moved to London in 1876 and became the Lord Chancellor's Visitor in Lunacy, a post he held until 1922. This was a well paid and highly prestigious job: one he secured against the competition of Henry Maudsley, the most illustrious psychiatrist of the day, who founded the Maudsley Hospital. But Crichton-Browne had abandoned research, and became a portentous public figure who worked to good effect in improving lighting, sanitation, and many public health problems. As an impressive after dinner speaker and radio broadcaster he kept himself prominent in the public eye; a favourite subject was his opposition to teetotalism, maintaining that "no writer has done much without alcohol".

Well versed in the great poets and novelists, his writings are a joy to read. ${ }^{3}$ He published an acclaimed book on Robert Burns, and a five volume autobiography. He met Thomas Carlyle and his family in London and after Carlyle's death he wrote extensively about him and his wife Jane Carlyle; he entered the seething controversy initiated by Froude, their biographer, about their personal lives and behaviour, ${ }^{5}$ which would now fill the tabloids for weeks.

Crichton-Browne assisted Charles Darwin with drawings and pictures when Darwin was writing his Expression of the emotions in man and animals. Such was Darwin's regard for him that he proposed his election to the Royal Society in 1883. Queen Victoria bestowed a knighthood in 1886. He lived until 1937, publishing his last book in his, that, year. In 1865, he had married Emily, youngest daughter of Dr J Halliday, a surgeon in Seacombe, Cheshire. She died in 1903 leaving a son and a daughter. His second wife Emily, was daughter of General Sir E Bulwer, and a great-niece of Bulwer-Lytton (Edward BulwerLytton, writer (1803-1873), who coined the memorable adage, "A good heart is better than all the heads in the world").

A portrait of Crichton-Browne by Hannah Gluckstein, 1928, is catalogued at the National Portrait Gallery. And you may not wish to learn that he was portrayed on a cigarette card in a series on Famous Scots published by Mitchell, Ardath in 1935.

His recipe for longevity was-“work and plenty of it".

J M S Pearce 304 Beverley Road, Anlaby, Hull HU10 7BG; imspearce@freenet.co.uk

\section{References}

1 Neve $M$, Turner T. What the doctor thought and did: Sir James Crichton-Browne 1840-1938. Medical History 1995;39:399-432.

2 Shorvon SD, Sander JW. The treatment of epilepsy at the National Hospital Queen Square, 1857-1939: a mirror of the first phase of the modern history of medical and surgical therapy In: Shorvon SD, Dreifuss FE, Fish DR, et al, eds. The treatment of epilepsy. Blackwell Science, Oxford, 1996.

3 Crichton-Browne J. The story of brain. Edinburgh and London: Oliver and Boyd, 1924.

4 Crichton Browne J. Stray leaves from a physician's portfolio. London: Hodder and Stoughton, 1927.

5 Crichton-Browne J. The nemesis of Froude: a rejoinder to J A Froude's "My Relations with Carlyle". London and New York: John Lane, 1903. 